\title{
Infinite Freedom of Space-Time for Zero-Energy-Entity in Quantum Mechanics
}

\author{
Chun T. Rim \\ GIST (Gwangju Institute of Science and Technology), Gwangju, Korea \\ Email:ctrim@kaist.ac.kr
}

How to cite this paper: Rim, C.T. (2018) Infinite Freedom of Space-Time for $\mathrm{Ze}$ ro-Energy-Entity in Quantum Mechanics. Journal of Modern Physics, 9, 525-531. https://doi.org/10.4236/jmp.2018.94036

Received: January 3, 2018

Accepted: March 6, 2018

Published: March 9, 2018

Copyright $\odot 2018$ by author and Scientific Research Publishing Inc. This work is licensed under the Creative Commons Attribution International License (CC BY 4.0).

http://creativecommons.org/licenses/by/4.0/

\section{c) (i) Open Access}

\begin{abstract}
Zero-energy state is investigated by taking infinitesimal energy and observing its uncertainty in space-time, adopting quantum mechanics. In this paper, the uncertainty in conventional quantum mechanics is found to be interpreted as freedom in space-time, which results in possibility of time travel and space transition of the zero-energy state, which could be information or mind. The wave function of a physical system composed of multiple particles or wave-packets is examined and found that it can be arbitrarily changed by grouping by observers. It leads to an idea that even infinitesimal energy or wave-packets in a heavy physical system may separately exist and it has the infinite freedom of space-time.
\end{abstract}

\section{Keywords}

Infinite Freedom of Space-Time, Zero-Energy Quantum Mechanics, Bipolar Quantum Information

\section{Introduction}

In non-relativistic quantum mechanics, the state of a physical system in space and time is represented by the wave function $\psi(\boldsymbol{x}, t)$, which is governed by the Schrödinger equation [1]:

$$
\left(i \hbar \frac{\partial}{\partial t}+\frac{1}{2 m} \nabla^{2}-V(\boldsymbol{x}, t)\right) \psi(\boldsymbol{x}, t)=0,
$$

where, $\hbar$ is the reduced Planck's constant $h /(2 \pi) ; m$ is the mass; and $V(x, t)$ is the potential of the system at three dimensional position $\boldsymbol{x}$ and time $t$. Note that (1) is linear in space and time so far as $m$ and $V(x, t)$ are not a function of $\psi(x, t)$, which is quite often assumed or approximated in quantum mechanics.

On the other hand, there is a fundamental limit to the precision of comple- 
mentary variables of a particle such as momentum $p$ and position $x$ as well as energy $E$ and time $t$, which is governed by Heisenberg's uncertainty principle [2]:

$$
\begin{gathered}
\sigma_{p} \sigma_{x} \geq \frac{\hbar}{2} \\
\sigma_{E} \sigma_{t} \geq \frac{\hbar}{2},
\end{gathered}
$$

where, $\sigma_{p}, \sigma_{x}, \sigma_{E}$, and $\sigma_{t}$ are the standard deviation of momentum, position, energy, and time, respectively. Though there are a few disputes on Equation (2) in literatures [3], the validity of Equation (2) is basically unchanged. Heisenberg uncertainty principle is so general that it can be applicable to a particle (or the wave-packet of a particle) of arbitrary mass $m$, arbitrary velocity $v(v \ll c)$, and arbitrary size.

So far, conventional quantum mechanics only have dealt with the physical system of non-zero mass or energy, as evidently identified from Equations (1) and (2). It is inevitable for the modern physics to deal with such a case because only measurable quantities are allowed to enter the scope of scientific research. We are living, however, in the world of information and mind consists of bits and neurons. The universe is not necessarily composed of physical entities with non-zero energy only, but also composed of information entities with zero- or near-zero-energy, which cannot be measured experimentally, but can only be logically proven with quantum information conservation.

An idea of dealing with zero-energy-entity was proposed, where zero-energy bipolar quantum entanglement can be non-local [4] [5] [6] [7]. Schrödinger equation was linked to the idea to explain that zero energy information can travel instantly [8]. Though not easy to demonstrate the bipolar quantum entanglement experimentally, this idea provides us the possibility of dealing with zero-energy entity not as artificial one but as physical one.

The speed of quantum information was measured by a Bell experiment [9], where nonlocal correlations were observed and Einstein's spooky action at a distance propagates much faster than at speeds of light by at least four orders of magnitude.

The above ideas confirmed the non-locality of nature in general and have stimulated the possibility of infinite freedom in space and time for the zero-energy-entity.

In this paper, the characteristics of entities with zero energy are explored in general form as an extension of quantum mechanics without the engagement of bipolar quantum entanglement. The zero-energy properties are gained from a physical system, which is composed of non-zero mass or energy. The multi-layered wave function is first introduced to explain the proposed idea. It is identified that infinite "freedom" of space and time is one of the characteristics of the zero-energy-entity. 


\section{Multi-Layered Wave Functions}

The wave function of a physical system composed of multiple particles, which are also represented by wave-packets, can be arbitrarily changed by grouping of observers. As shown in Figure 1, a physical system, represented as the dotted square box, contains $n$ particles of different momentum and energy $\left(\boldsymbol{p}_{k}, E_{k}\right)$ for the $k$-th particle. The wave function of a group of particles, called "group wave function", is the superposition of individual wave function $\psi_{k}(x, t)$ as follows:

$$
\begin{aligned}
& \psi_{A}(\boldsymbol{x}, t)=\sum_{k \in A} \psi_{k}(\boldsymbol{x}, t) \\
& \psi_{B}(\boldsymbol{x}, t)=\sum_{k \in B} \psi_{k}(\boldsymbol{x}, t) .
\end{aligned}
$$

In Equation (3), the wave function $\psi_{k}(x, t)$ is determined by the following generalized Schrödinger equation, which is applicable even to the non-linear case where the particles interact each other and the potential $V_{k}\left(\psi_{i}, \boldsymbol{x}, t\right)$ is a function of wave functions $\left\{\psi_{i}(x, t) \mid i=1,2, \cdots, n\right\}$ :

$$
\left(i \hbar \frac{\partial}{\partial t}+\frac{c^{2}}{2 E_{k}} \nabla^{2}-V_{k}\left(\psi_{i}, \boldsymbol{x}, t\right)\right) \psi_{k}(\boldsymbol{x}, t)=0 ; \quad k=1,2, \cdots, n .
$$

An example case of the non-linear Schrödinger equation is the free electrons in a metal lattice, where each electron interacts not only with each other but also with positrons in nuclei. In Equation (4), the energy of the $k$-th particle $E_{k}$ is introduced instead of the mass $m_{k}$ for the general expression purpose as follows:

$$
E_{k}=m_{k} c^{2} ; \quad k=1,2, \cdots, n \text {. }
$$

In the case that the wave function of each particle $\psi_{k}(\boldsymbol{x}, t)$ is independent each other, e.g., the ideal gas where no interaction exists between each particle, Equation (4) becomes as follows:

$$
\left(i \hbar \frac{\partial}{\partial t}+\frac{c^{2}}{2 E_{k}} \nabla^{2}-V_{k}(x, t)\right) \psi_{k}(x, t)=0 ; \quad k=1,2, \cdots, n .
$$

Note from Equation (3) that the selection of particles, i.e., grouping, can be

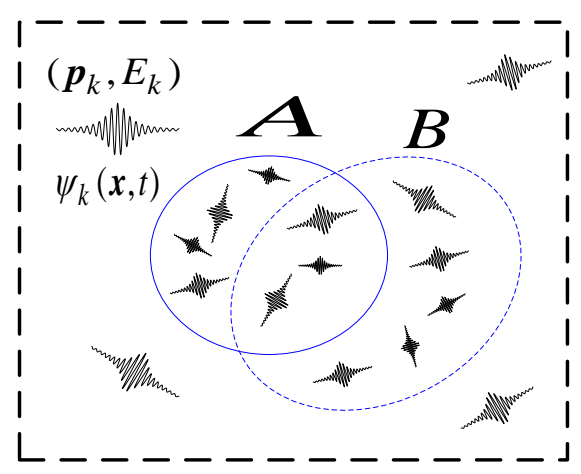

Figure 1. A physical system composed of multiple wave-packets of particles, groups $A$ and $B$ are arbitrary. 
arbitrarily made by observers. This means that the group wave function changes as the observer just "thinks" of the boundary of a group. The grouping is concerned with "measurement" of particles, where the boundary of a group corresponds to the scope of measurement.

This grouping idea can be extended to a layered physical system consists of different physical properties, e.g., a group of light particles (such as free electrons) and a group of heavy particles (such as neutrons), as shown in Figure 2. If there is no interaction between two groups $A$ and $B$ as well as between each particle in a group, there is no doubt that the group wave function of Equation (3) is determined by the superposition of individual wave function of Equation (6).

In the case that there is any interaction between each particle or each sub-system, as depicted by arrows in Figure 2, the superposition is still valid if the generalized Schrödinger equation of Equation (4) is used to obtain the non-linear individual wave functions. In other words, the group wave function is simply the sum of individual wave functions, which could be obtained from either linear or non-linear Schrödinger equations. This apparently strange statement can be understood from a similar case that the total energy of a particle can be obtained by the sum of potential energy and kinetic energy, which may be non-linear with respect to the velocity or position of the particle. The energy is linear with respect to each energy, but each energy can be non-linear with respect to other variables.

It can be still argued that the wave function may not be linear each other under some extreme conditions; however, it is not likely happen to conventional wave packets, and it will beyond the scope of this paper if it is the unusual case.

\section{Space-Time Uncertainty of Infinitesimal Energy}

Now the multi-layered wave function concept is extended to the zero-energy-entity. As shown in Figure 3, the "bit" of information can be understood as the energy-entity of zero value. The value of information " 0 " or " 1 " is not directly related to the energy state of the information hardware. The information itself may have zero energy whereas the information hardware should have non-zero energy as identified from Shannon's Theorem in conventional

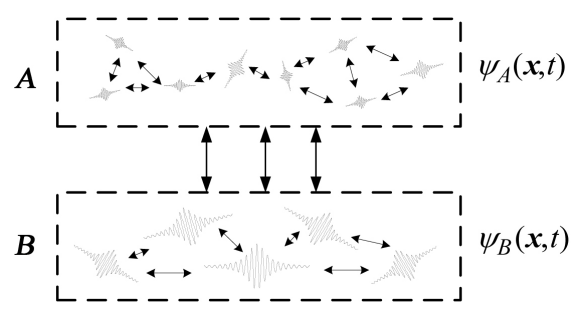

Figure 2. A layered physical system composed of sub-physical system containing multiple wave-packets of particles, where the group $A$ has light particles and the group $B$ has heavy particles, for example. 


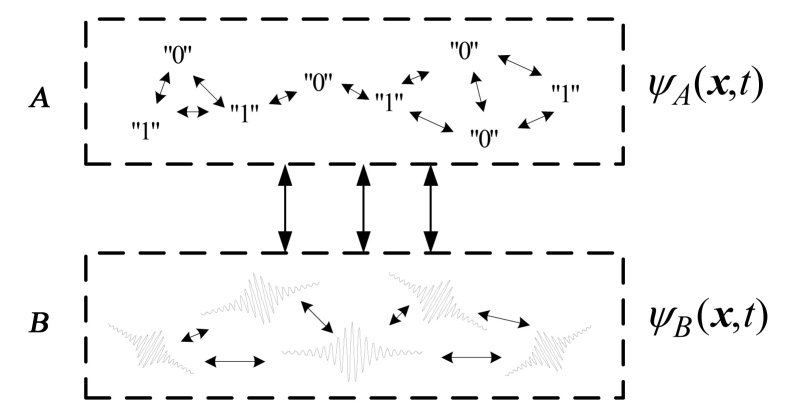

Figure 3. Information of bits represented as the wave functions of zero-energy-entity (Group $A$ ), which are created by a hardware system (Group B).

communication systems [10]. As evidently identified from Equations (4) or (6), the Schrödinger equations cannot be directly applied to the characterization of the zero-energy-entity because the denominator of energy term becomes zero.

It is remarkable that the Schrödinger equations of Equation (1) or (4) are applicable to any physical systems such as quarks, electrons, neutrons, atoms, molecules, and a car in theory. In other words, the Schrödinger equation itself is irrelevant to the energy level and size of any specific particles or material. Therefore, the Schrödinger equation is applicable to a virtual wave packet with tiny energy, which is much less than a quark. There is no energy limit in the application of the Schrödinger equation, i.e., the applicable energy level can be much less than the Planck's constant.

Hence, the Schrödinger equation is applicable to infinitesimal energy packet because it is still in the region of non-zero energy though its limiting value is zero. The question is "what is the difference between 'exactly zero energy' and 'asymptotically zero energy'?". If only the asymptotical behavior of zero-energy is examined instead of that of truly zero-energy, the Schrödinger equation can be credibly used for this purpose.

Still a remaining question is that "what is the nature of information?". The information, defined as a group of wave functions in this paper, may be either a truly zero-energy-entity or an asymptotically zero-energy-entity. Because we have neither a theory nor a measurement method to deal with the truly zero-energy-entity in quantum mechanics, it is beyond the scope of this paper. Instead, it is postulated in this paper that the information is an asymptotically zero-energy-entity. Under this assumption, the behaviors and characteristics of information can be examined within the scope of quantum mechanics in this paper.

Because Schrödinger equation is applicable to infinitesimal artificial wave packet, Heisenberg uncertainty principle is applicable to an artificial wave-packet. From Equation (2), the uncertainties in position and time of an infinitesimal wave packet can be determined as follows:

$$
\lim _{\sigma_{p} \rightarrow 0} \sigma_{x} \geq \lim _{\sigma_{p} \rightarrow 0} \frac{\hbar}{2 \sigma_{p}}=\infty
$$




$$
\lim _{\sigma_{E} \rightarrow 0} \sigma_{t} \geq \lim _{\sigma_{E} \rightarrow 0} \frac{\hbar}{2 \sigma_{E}}=\infty,
$$

where, the limiting values of $\sigma_{p}$ and $\sigma_{E}$ are both zero because the limiting values of mass and energy of an infinitesimal wave packet are zero. As identified from Equation (7), the uncertainties of information in position and time are infinity. As the wave function represents the probability of existence, information can be measured everywhere and anytime in universe.

Therefore, it can be said that the asymptotically zero-energy-entity such as information and mind has infinite "freedom" of space and time in quantum mechanics. Zero mass or zero energy gives rise to the freedom of going everywhere and anytime. In theory, information and mind may reach to any place and time in universe at once. In other words, a time machine or a teleporter can be realized by information machines (computers or artificial intelligence) or living things including human being, which can think and therefore has mind. This result is consistent to the bipolar atom theory, which is applicable to not only physical worlds but also logical, mental, social, and biological worlds [4] [5] [6] [7] [8].

Note that the zero-energy-entity (Group $A$ ) is based on and correlated with the non-zero-energy-entity (Group $B$ ), as shown in Figure 3. Therefore, the non-zero-energy-entity such as a computer and human body cannot be a time machine or teleporter but information and mind can be.

\section{Conclusion}

Zero-energy-entity such as information and mind is found, in this paper, to have infinite freedom of space and time. This conclusion is identical to the idea of zero-energy bipolar quantum entanglement. The proposed theory in this paper, however, no longer relies on physical systems having non-zero energy to get the zero-energy-entity property. By grouping method, applied to Schrödinger equation, wave functions of zero-energy-entity can be separately dealt with from that of non-zero-energy-entity. Thus, it is found from Heisenberg's uncertainty principle that infinitesimal energy-entity has infinite uncertainty in position and time. In theory, a time machine or a teleporter can be realized by an information machine or a living thing which has mind. The measurement and detection of such information and mind in quantum mechanics is left for future works. Bipolar relativity [4] [5] [6] [7] [8] could be a possible way of proving the proposed idea in this paper.

\section{References}

[1] Schrödinger, E. (1926) Physical Review, 28, 1049. https://doi.org/10.1103/PhysRev.28.1049

[2] Kennard, E.H. (1927) Zeitschrift für Physik, 44, 326. (In German)

[3] Ozawa, M. (2003) Physical Review A, 67, Article ID: 042105.

[4] Zhang, W.-R. (2011) Yinyang Bipolar Relativity: A Unifying Theory of Nature, Agents and Causality with Applications in Quantum Computing. Cognitive Infor- 
matics and Life Sciences, Book. https://doi.org/10.4018/978-1-60960-525-4

[5] Zhang, W.-R. (2012) Journal of Modern Physics, 3, 1261-1271.

https://doi.org/10.4236/jmp.2012.329163

[6] Zhang, W.-R. (2013) Journal of Quantum Information Science, 3, 93-105. https://doi.org/10.4236/jqis.2013.32014

[7] Zhang, W.-R. (2017) IEEE Transactions on Fuzzy Systems, PP, No. 99. http://ieeexplore.ieee.org/document/7886348/

[8] Zhang, W.-R. (2016) Journal of Quantum Information Science, 6, 62-97. https://doi.org/10.4236/jqis.2016.62008

[9] Salart, D., Baas, A., Branciard, C., Gisin, N. and Zbinden, H. (2008) Nature, 454, 861-864. https://doi.org/10.1038/nature07121

[10] Shannon, C.E. (1962) The Mathematical Theory of Communication. Urbana University of Illinois Press, Urbana. 